\title{
Fabrication of Micro-grating Structure by Nanosecond Laser
}

\author{
Yanlong Huang, Jianguo Zhu* \\ Faculty of Civil Engineering and Mechanics, Jiangsu University, Zhenjiang, China
}

Email address:

hll_1314@126.com (Yanlong Huang), zhujg@ujs.edu.cn (Jianguo Zhu)

\section{To cite this article:}

Yanlong Huang, Jianguo Zhu. Fabrication of Micro-grating Structure by Nanosecond Laser. Optics. Special Issue: Optical Techniques for Deformation, Structure and Shape Evaluation. Vol. 4, No. 3-1, 2015, pp. 5-8. doi: 10.11648/j.optics.s.2015040301.12

\begin{abstract}
The fabrication method by nanosecond laser technology for micro-grating is investigated in this paper. A nanosecond laser system is established and the mechanism of laser processing on material surface is theoretically analyzed. The damage threshold of stainless steel is obtained experimentally when the nanosecond laser irradiates on the specimen's surface and the influence of the laser pulse energy to the fabricated micro-gratings are also studied. In the end, micro-gratings with high-quality are provided to validate the feasibility of the fabrication method by nanosecond laser technology.
\end{abstract}

Keywords: Grating, Nanosecond Laser, Photomechanics

\section{Introduction}

Gratings are straight, parallel, and equispaced grooves. As a basic optical component, it can be used in various optical technologies, such as moiré interferometry [1], Geometric Phase Analysis (GPA) method [2] and various microscopic moiré methods [3], to measure the surface deformation on the object. Currently grating fabrication methods include holographic lithography method [4], nanoimprint lithography method [5], electron beam lithography (EBL) method [6], and focused ion beam (FIB) etching method [7], etc. Holographic lithography method using an interference of two coherent beams and exposure of the photoresist under the interference path to fabricate the grating, However, the complex arrangement of optical components has made it very difficult to put into practice, and has accordingly restrained its wide application. Nanoimprint lithography method requires a template to fabricate the grating. EBL and FIB etching belong to the direct writing technology that can be fabricated micro- and nano- gratings, however, low throughput and high cost make them impractical for commercialization.

As a major achievement of the 20th century science and technology, laser has been widely used due to its properties such as high peak power, strong direction, and good coherence. The energy density of nanosecond laser is very high ( 1J), and recently it has been used to fabricate microstructures directly on the surface of materials such as metals, semiconductors, ceramics [8], etc. Also, the nanosecond laser can fabricated micro-gratings on the surface of the specimen without mask, which provides an alternative way of grating fabrication and its corresponding research is of significant importance.

\section{Nanosecond Laser Processing [9]}

When a high-energy laser beam irradiates to a material, the temperature of spotted surface increases sharply due to the absorption of the laser energy. The material begins to melt after the temperature of the spotted area reaches its melting point. And the material begins to evaporate if the temperature continues rising and reaches the latent heat of material ablation with further absorption of laser energy. Generally, there are three regions when the high-energy laser irradiates to the material, as shown in Fig. 1. 1) A plasma region with high temperature and density (Pla) formed on the surface of the material; 2) A melting center region of the spotted material (Mel); 3) A heat affected zone (HAZ) under the Mel region. 


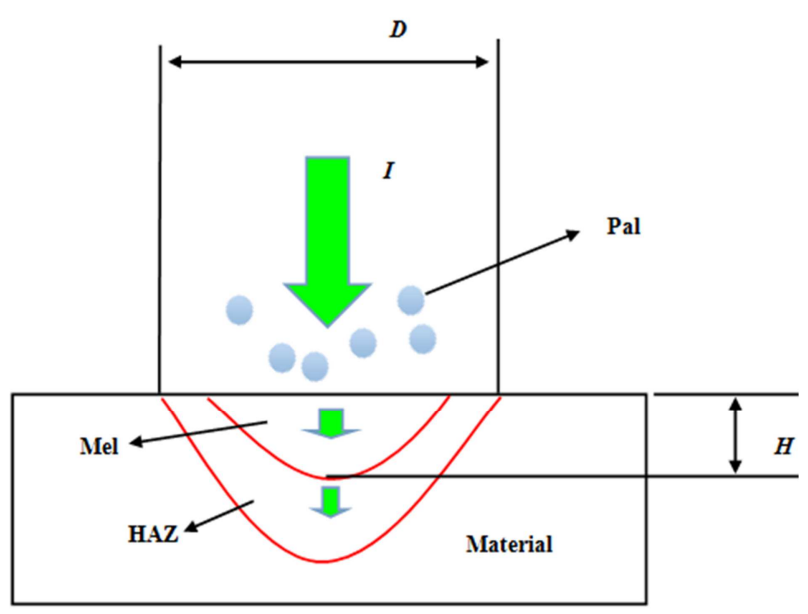

Figure 1. Schematic diagram of principle of nanosecond laser processing.

The damage threshold of a material irradiated by a laser beam is one of its intrinsic properties. When a single-pulse laser irradiates on a material, the damage of the material is mainly associated with the energy of laser. The relationship between the laser intensity $\left(I_{0}\right)$ on the surface of the specimen and its single-pulse energy $E$ is [10]:

$$
I_{0}=\frac{2 E}{\pi \omega_{0}^{2}}
$$

where $\omega_{0}$ is the waist radius of the laser beam $(\mu \mathrm{m})$. And the etching diameter can be determined as

$$
D^{2}=2 \omega_{0}^{2} \ln \left(\frac{\mathrm{I}_{0}}{\mathrm{I}_{\mathrm{th}}}\right)
$$

where $D$ indicates the etching diameter and $I_{t h}$ is the threshold of energy density $\left(\mathrm{J} / \mathrm{cm}^{2}\right)$. Substituting Eq. (1) into Eq. (2), we can get Eq. (3)

$$
D^{2}=2 \omega_{0}^{2} \ln E+2 \omega_{0}^{2} \ln \left(\frac{2}{\pi \omega_{0}^{2} I_{t h}}\right)
$$

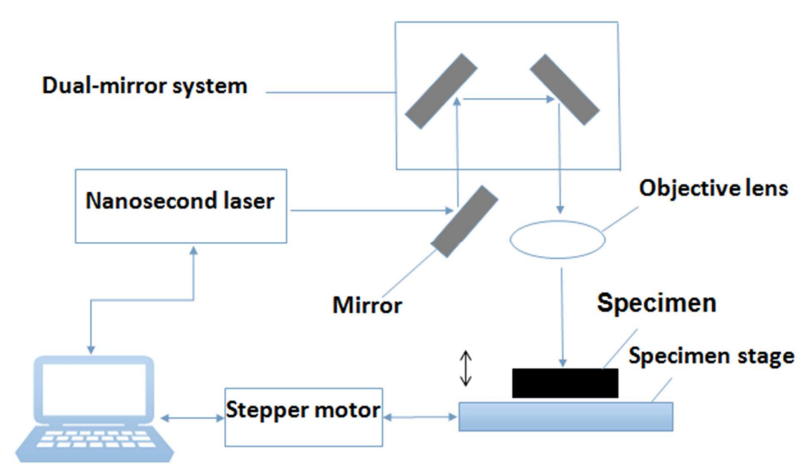

Figure 2. Schematic diagram of a nanosecond laser processing system

Fig. 2 is a schematic diagram of a nanosecond laser processing system (Continuum) with wavelength of $532 \mathrm{~nm}$, pulse width of 500ns, pulse frequency of $1 \mathrm{KHz}$, and maximum pulse energy of $1 \mathrm{~J}$. The laser beam path is adjusted by a dual-mirror system which is controlled by a computer. Note that software was developed to move the beam spot on the surface precisely. The specimen stage is controlled by a stepping motor and can move vertically, thus the specimen can be focused by the laser beam through an objective lens.

\section{Experiments and Results}

\subsection{Experiments}

The specimen was 304 stainless steel $(50 \mathrm{~mm} \times 50 \mathrm{~mm} \times$ $5 \mathrm{~mm}$ ). The specimen surface was ground step by step with waterproof abrasive papers (180 \#, 400 \#, 800 \#, 1200 \#, $2000 \#)$, and then polished by grinding paste. Finally, we used GH4169 and $\mathrm{Si}$ as substrate materials to fabricate gratings.

In order to obtain the etching parameters when a single-pulse laser beam irradiates on the specimen, lines were etched by the nanosecond laser system with different pulse energy ranging from $4.9 \mu \mathrm{J}$ to $80 \mu \mathrm{J}$. Micro-structural morphology and widths of the etched areas were observed and measured by an optical microscopy. In the end, suitable parameters were chosen to fabricate micro-scale gratings.

\subsection{Results and Discussion}

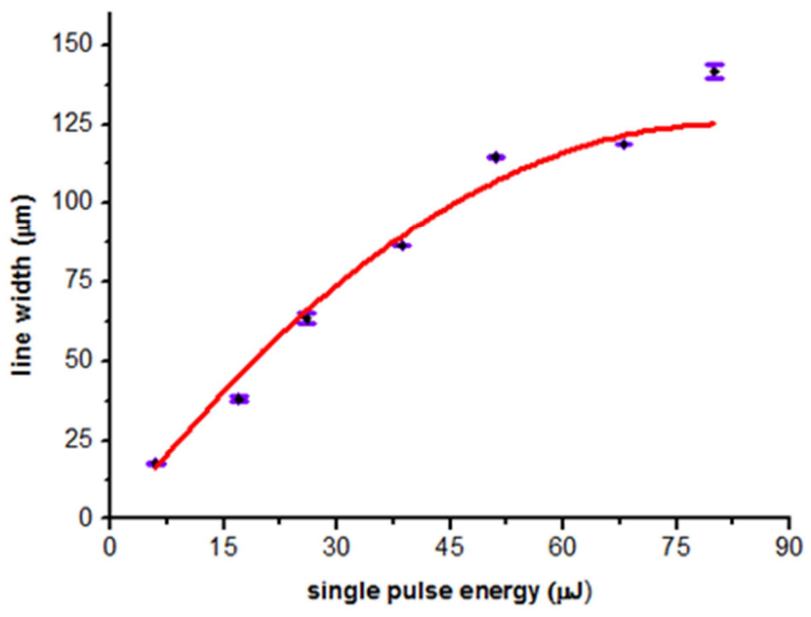

(a)

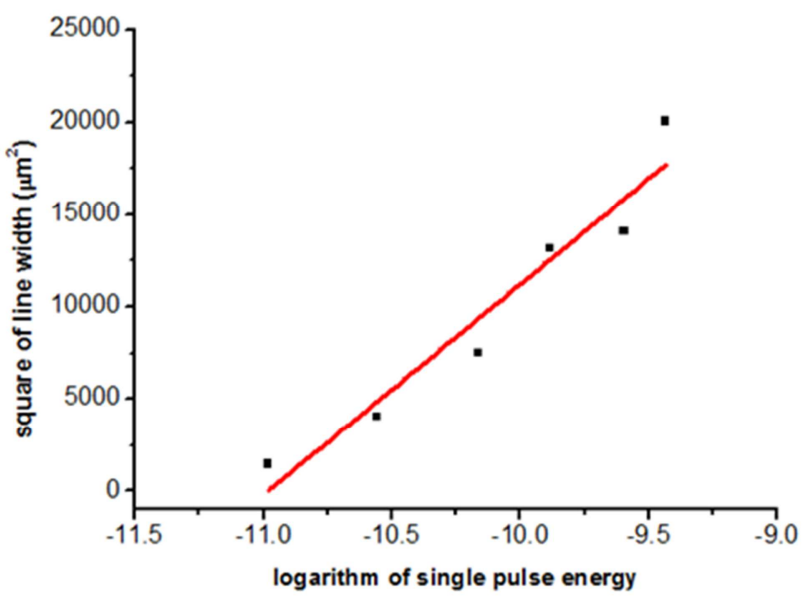

(b)

Figure 3. (a) Relation curve of line width and pulse energy (b) Relation curve of square of line width and logarithm of the single pulse energy. 
Fig. 3(a) illustrates the variation in the line width with the pulse energy. According to Eq. (3) the single pulse energy $E$ and the line width $D$ have a non-linear relationship. It is observed that the line width increases with the single pulse energy. The line width changes obviously in low energy situations, and the line width changes smoothly with the increase of energy. In high energy situations, energy is concentrated relatively and the HAZ is small, that is why the line width changes smoothly. Fig. 3(b) illustrates the variation in the square of the line width with logarithm of the single pulse energy. Straight line was fitted out based on these data which is consistent with Eq. (3). It can be calculated that the slope is $2 \omega_{0}{ }^{2}=11442.8 \mu \mathrm{m}^{2}$, thus, the waist radius of the laser beam is $75.6 \mu \mathrm{m}$. Also, the pulse energy threshold can be calculated when letting $D=0 \mu \mathrm{m}$, which is $E_{t h}=17 \mu \mathrm{J}$. Therefore, the threshold of energy density $I_{t h}=0.189 \mathrm{~J} / \mathrm{cm}^{2}$ according to Eq. (1).

Micro-scale gratings were fabricated based on the above parameter studied. The specimen surface will not have etched trace, if the nanosecond laser pulse energy is less than the damage threshold. However, if the nanosecond laser pulse energy is greater than the damage threshold, microstructures will appear on the specimen surface. And the quality of microstructures changes with the nanosecond laser energy. Hence, we need to choose suitable laser pulse energy according to damage threshold.

Fig.4 shows optical images of gratings using laser pulse energy of $51 \mu \mathrm{J}$, a constant scanning speed of $10 \mathrm{~mm} / \mathrm{s}$. Fig. 4(a) shows the image of a horizontal grating and its average pitch is about $30 \mu \mathrm{m}$. A good quality of grating is shown in the etched region. The lower pitch (about $25 \mu \mathrm{m}$ ) of grating is shown in Fig. 4(b). The overlap occurs between grating strips. The quality of grating is poor and the structure is not clear. Fig. 5 shows the SEM image of the grating of Fig. 4(a). It is observed that grating strips is clearly, and the distorted nanostructures were formed.

Fig.6 shows the distribution of Gaussian energy density in respect to the distance. The two curves in the left don't have phenomenon of overlap above line of ablation. The distance between the peaks of them is the pitch of grating. In this case the grating has a relatively low frequency. Fig. 4(a) has the same situation with the phenomenon. The three curves in the right have phenomenon of overlap above line of ablation. This situation will affect the clarity of microstructure; Fig. 4(b) has the same situation with the phenomenon. Therefore, higher frequency of grating is limited by the laser spatial resolution, when the nanosecond laser direct writing technology is adopted to fabricate micro-grating. Otherwise, there will be appearing overlap and discontinuous phenomenon.

Fig.7 shows the different gratings that fabricated by nanosecond laser direct writing technology in different substrate materials. The substrate material is GH4169 and its average pitch is about $30 \mu \mathrm{m}$ in Fig. 7(a). It is difficult to fabrication than stainless steel, because its density is relatively large. The quality of grating is poor and the structure is not clear. The substrate material is $\mathrm{Si}$ and its average pitch is about $30 \mu \mathrm{m}$ in Fig. 7(a). We can easily process in the material surface and the grating is good.

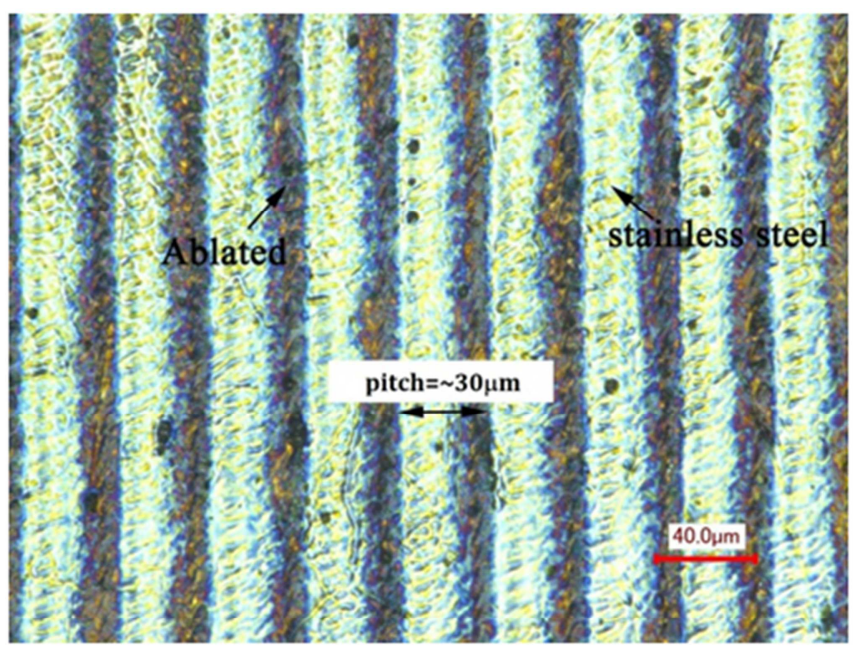

(a)

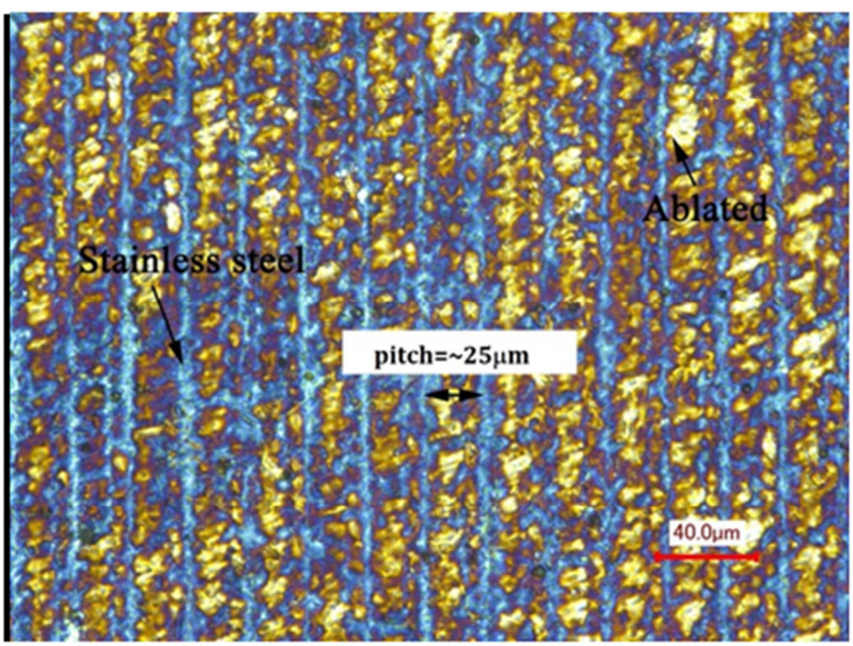

(b)

Figure 4. Optical images of gratings (a) pitch $=\sim 30 \mu \mathrm{m}$ and (b) pitch $=\sim 25$ $\mu m$

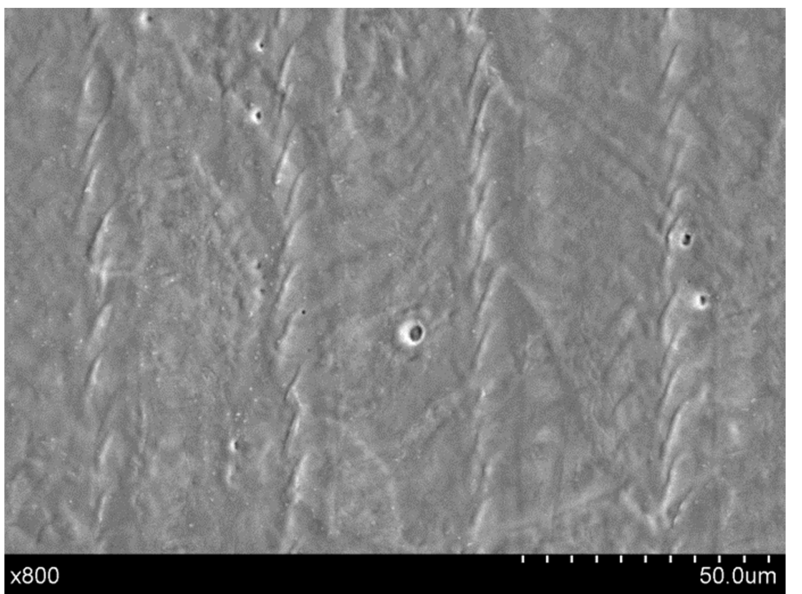

Figure 5. SEM image of grating (pitch $=\sim 30 \mu \mathrm{m})$ 


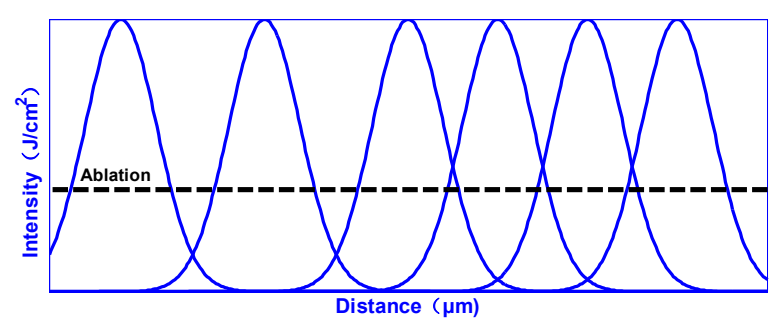

Figure 6. The distribution of Gaussian energy density along with the distance

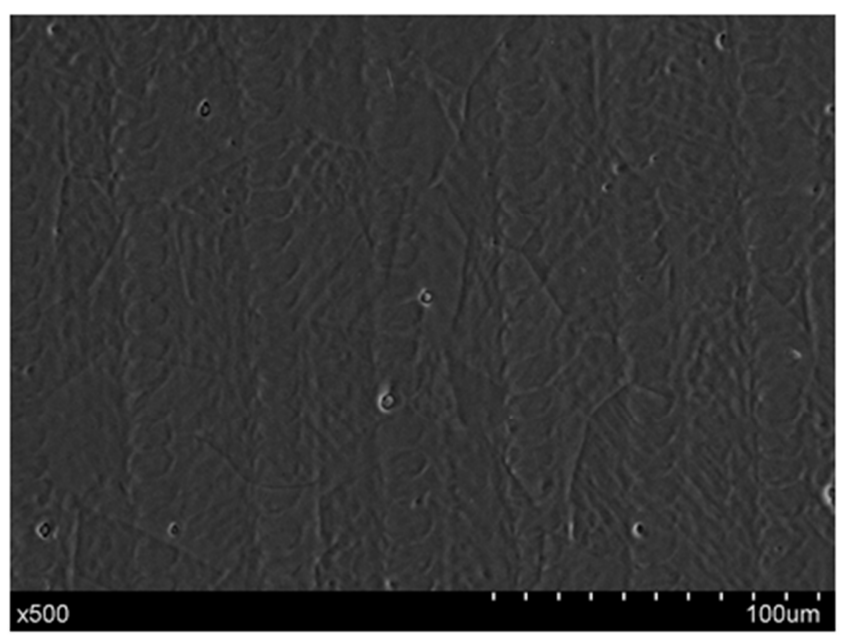

(a)

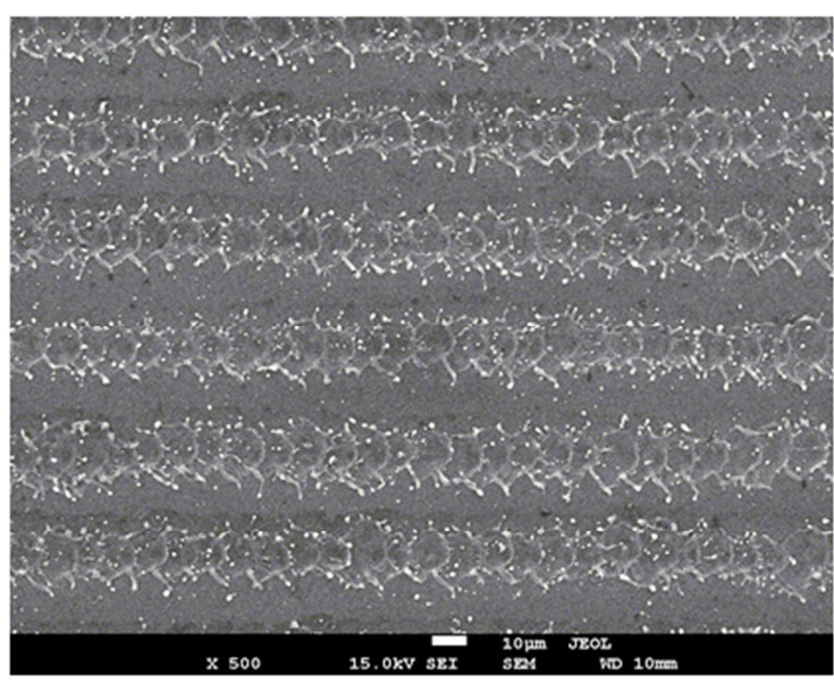

(b)

Figure 7. SEM images of gratings (pitch $=\sim 30 \mu \mathrm{m}$ ) (a) GH4169 and (b) $\mathrm{Si}$

\section{Conclusions}

In this paper, a nanosecond laser system was established and the fabrication method for micro-grating was studied. The mechanism of laser processing on material surface was theoretically analyzed. The damage threshold $\left(I_{t h}=0.189 \mathrm{~J} / \mathrm{cm}^{2}\right)$ and laser beam waist radius $\left(\omega_{0}=75.6 \mu \mathrm{m}\right)$ with respect to stainless steel were derived by experiments. The experimental results have demonstrated clear grating with low pitch $(\sim 30$ $\mu \mathrm{m})$ can be fabricated using this method. The difficulties in the fabrication of high frequency grating by nanosecond laser direct writing are analyzed. This method of grating fabrication is faster and more convenient than other methods. Moreover, the fabrication of high frequency grating needs further researches.

\section{Acknowledgements}

The authors are grateful to the financial support by the National Natural Science Foundation of China (Grant Nos. 11232008 and 11372118).

\section{References}

[1] B.Han, "Higher sensitivity moiré interferometry for micromechanics Studies," Optical Engineering vol. 31, pp.1517-1526, 1992.

[2] J.L.Rouviere, E.Sarigiannidou. Theoretical discussions on the geometrical phase analysis. Ultramicroscopy 106(2005)1-17.

[3] Xie Huimin, Shang Haixia, Dai Fulong, et al. "Phase shifting SEM moiré method," Optics and Laser Technology, vol.36(4), pp.291-297, 2004.

[4] Moulart R, Rotinat R, Pierron F, et al. "On the realization of microscopic grids for local strain measurement by direct interferometric photolithography,"Optics and lasers in engineering, vol.45(12), pp.1131-1147, 2007.

[5] Tang M, Xie Huimin, Li Y, et al. "A New Grating Fabrication Technique on Metal Films Using UV-Nanoimprint Lithography," Chinese Physics Letters, vol.29(9), pp.098101,2012.

[6] Kishimoto S, Tanaka Y, Yin F, et al. "Strain distribution measurement in laminated martensitic/austenitic steel during a compressive test by the electron moire method," Journal of Strain Analysis for Engineering Design, 2011.

[7] Du Hua, Xie Huimin, Guo Zhiqiang, et al. "Development of the Technique for Fabricating Submicron MoiréGratings on Metal Materials Using Focused Ion Beam Milling," Chinese Physics Letters, vol.24,pp.2521-2524, 2007.

[8] Yung-Chun Lee, Chun-Ming Chen, Chun-Ying Wu. "A new excimer laser micromachining method for axially symmetric 3D micro-structures with continuous surface profiles," Sensors and Actuators A. vol.117,pp.349-355, 2005.

[9] Felter T E, Hrubesh L, Kubota A, et al. "Laser damage probability studies of fused silica modified by $\mathrm{MeV}$ ion implantation," Nuclear Instruments and Methods in Physics Research B, vol. 27,pp.72-79, 2003.

[10] J.M.Liu. "Simpletechnique for measurements of pulsed Gaussian-beam spot sizes,” Opt. Lett., vol.7(5), pp.196 198, 1982. 